\title{
Profile of Anti-Chlamydia \\ Immune Responses in \\ Complicated (Infertile) and Non-complicated (Fertile) Genital Infections
}

\author{
Joseph U Igietseme ${ }^{1,2 *}$, Caroline Ukwade ${ }^{3}$, Sander Ouburg ${ }^{4}$, \\ Yusuf Omosun ${ }^{1,2}$, Kahaliah Joseph ${ }^{1}$, James Partin ${ }^{1}$, Qing \\ $\mathrm{He}^{1,2}$, Francis O Eko'2, Debra Ellerson', Claudiu Bandea', \\ Servaas A Morré ${ }^{4,5}$, Guangming Zhong ${ }^{6}$, Osaretin Albert T \\ Ebuehi $^{3}$ and Carolyn M Black ${ }^{1}$
}

${ }^{1}$ Centers for Disease Control and Prevention (CDC), Atlanta, GA, USA

${ }^{2}$ Morehouse School of Medicine, Atlanta, GA, USA

${ }^{3}$ Department of Biochemistry, University of Lagos, Lagos, Nigeria

${ }^{4}$ Laboratory of Immunogenetics, Department of Medical Microbiology and Infection Control, VU University Medical Centre, Amsterdam, The Netherlands

5Institute of Public Health Genomics, Department of Genetics and Cell Biology, Research Institute GROW, Faculty of Health, Medicine \& Life Sciences, University of Maastricht, Maastricht, The Netherlands

${ }^{6}$ Department of Microbiology and Immunology, University of Texas Health Science Center at San Antonio, San Antonio, TX 78229, USA

\begin{abstract}
The simultaneous induction of protective and immunopathogenic responses during genital $C$. trachomatis infection is proposed to respectively confer partial immunity and at least partly drive the complications such as pelvic inflammatory disease and tubal factor infertility. T cell-mediated immune response is crucial for chlamydial immunity in experimental animal models and in humans. However, the levels of T cell-derived cytokines in human specimens such as serum or mucosal tissue fluids can be influenced by several factors in addition to the incident infection. Therefore, it is uncertain whether T cell cytokine levels in biological fluids can predict infections that lead to complications, and can be used as reliable biomarkers of chlamydial disease complications, such as tubal factor infertility. Using a reliable murine model of chlamydiainduced infertility, we tested the hypothesis that the anti-chlamydial $T$ cell immune effectors induced during infections that produce complications are qualitatively or quantitatively distinct from
\end{abstract}

*Corresponding author: Joseph $\cup$ Igietseme, Centers for Disease Control and Prevention (CDC), 1600 Clifton Road, Mail Stop G-36, Atlanta, GA 30333, USA, Tel: +1 4046393352; Fax: +1 4047184011; E-mail: jigietseme@cdc.gov

Citation: Igietseme JU, Ukwade C, Ouburg S, Omosun Y, Joseph K, et al (2015) Profile of Anti-Chlamydia Immune Responses in Complicated (Infertile) and Non-complicated (Fertile) Genital Infections. J Clin Immunol Immunother 2: 006 .

Received: January 08, 2015; Accepted: January 25, 2015; Published: February 09, 2015 non-complicated infections. The results revealed that infected infertile mice exhibited lower systemic anti-chlamydial total IgG levels than animals with comparable microbiological shedding of chlamydiae but protected from infertility by treatment with the pan-caspase inhibitor Z-VAD-FMK. Interestingly, infected fertile mice showed greater Th1-type cytokines, mostly TNF- $\alpha$, IFN- $y$ and IL-17 than infertile animals, while levels of the Th2-type cytokines, IL-5 and IL-10 were higher in infected infertile mice than fertile mice. These profiles of systemic cytokine levels in mice are corroborated by clinical findings demonstrating that chlamydial infection with tubal pathologies elicited a predominantly Th2 immune response. Thus, antigen-specific $T$ cell cytokine response may distinguish infections leading to immunity versus sequelae, providing a useful prognostic biomarker and as a guide for vaccine design and evaluation.

Keywords: Chlamydia; Immune Profile; Infertility; Tubal Pathologies

\section{Introduction}

C. trachomatis genital infection is the most common bacterial Sexually-Transmitted Disease (STD) worldwide and in women Pelvic Inflammatory Disease (PID), ectopic pregnancy and infertility are serious complications that are of considerable public health concern. Quantitatively and qualitatively measurable humoral and $\mathrm{T}$ cell-mediated immune responses are associated with genital chlamydial infection; however, modulatory strategies to skew these responses toward protective immune effectors (antibodies, $\mathrm{T}$ cells and cytokines) mediating resolution of an infection or acquisition of immunity and protection from re-infection are among the challenging issues in the continuing effort to develop an efficacious chlamydial vaccine. Clinical studies in humans and experimentation in animal infection models have revealed that immunity to C. trachomatis correlates with a strong CD4 T helper type 1 (Th1) response and a complementary antibody response; the major role of anti-chlamydial antibody response is to foster a rapid and robust memory $\mathrm{T}$ Cell Mediated Immune (CMI) response during reinfections and possibly the neutralization of infectious particles [1-5]. Thus, as important immunologic correlates for vaccine testing and evaluation, a potentially efficacious vaccine should induce a strong CD4 Th1 response and accessory IgG and IgA antibodies in mucosal and systemic tissues.

The simultaneous and paradoxical induction of immunoprotective and immunopathogenic immune responses during chlamydial infection in humans and in experimental animals would suggest that at least some chlamydial antigens may induce both responses, or immunopathology only, as previously demonstrated [6-9], and therefore need to be "de-toxified" or remove from any potential future vaccine. Besides, the recent reports that certain cytokine profiles of anti-chlamydial immune effectors, are critical for preventing immunopathology [10,11] may offer appropriate screening methods for antigen immuno-toxicity or potential for inducing protective immunity; accordingly, the induction of an appropriate immune profile that include IFN- $\gamma$ or an inappropriate cytokine profile, such as the absence of IFN- $\gamma$ or a predominantly Th2 or T17 response [10-13], and presence of potentially pathogenic properties in the antigen [14-18] are promising vaccine antigen screening and discovery approaches. In addition, the profile of cytokines and immune effectors of an anti-chlamydial immune 
response in infections with and without pathologic complications may also provide a useful molecular and immunological biomarker for predicting chlamydial pathologies. In Chlamydia-susceptible hosts, the presence of the cryptic plasmid and the profile of cytokines produced by anti-chlamydial $\mathrm{T}$ cell lines and clones were important factors that determined the development of infection complications $[10,11,19,20]$. Besides, successful chlamydial infection in humans was attributed to the skewing to immune response to a predominantly Th2 type and low Th1 response [21]. The apparent biological and pathologic relationship between the profile of immune effectors and the outcome of chlamydial infection, led us to hypothesize that the profile of anti-chlamydial immune responses in hosts who resolved the infection without complications may be different from when the host suffers complications. To test this hypothesis, we analyzed the serum antibody and cytokine responses in an establish mouse model of Chlamydia-induced infertility in which anti-caspase treatment prevented the onset of infertility without affecting infectivity [19]. The results from immune profile analysis in animals suggested that chlamydial infection with tubal pathologies elicited a predominantly Th2 immune response, and infections without pathologies are likely to be predominantly Th1 type, providing a useful prognostic biomarker to also guide the design and evaluation of safe efficacious vaccines.

\section{Materials and Methods}

Chlamydia trachomatis stocks, animal infection and assessment of infertility. Stocks of C. trachomatis serovar L2 were propagated in HeLa cells and purified Elementary Bodies (EBs) were titered as Inclusion-Forming Units per milliliter (IFU/ml) by standard procedure [22]. Female C57BL/6 mice, 5-8 weeks old, were obtained from either Taconic Farms, Inc (Hudson, NY) and infected intravaginally with $1 \times 10^{5}$ IFU per mouse with chlamydiae while under the long-acting anesthetic sodium pentobarbital (30 ug/body weight) (Sigma-Aldrich, St Louis, MO) approximately 5 days after Intramuscular (IM) administration of $2.5 \mathrm{ug} /$ mouse Depo Provera (medroxyprogesterone Acetate; Pfizer Inc, NY, USA). These conditions are a key factor to a successful mouse model of chlamydial genital infection using the human chlamydial strains [23]. C. trachomatis serovar L2 was used in this study because the strain was used to establish the mouse infertility model we studied; and the availability of a naturally occurring plasmid-free strain of L2 facilitated comparative studies to identify the microbial virulence factors in other ongoing studies in the laboratory. Control mice were sham infected. The course of the infection was monitored by periodic (every 3 days) cervico-vaginal swabbing of individual mouse and tissue culture isolation and enumeration of chlamydial inclusions by a standard immunofluorescence method [22].

Chlamydia-induced infertility was assessed by infecting female mice twice at 4-6 wk intervals to ensure repeated infection that enhanced infertility [24] and mating them with proven fertile males, followed by monitoring daily weight-gain for approximately 21 days. A consistent 3 days of weight gain by mice was considered evidence of pregnancy. The numbers of pregnant mice and the mean number of embryos in the different groups were enumerated and calculated as the mean number of embryos per experimental group. In addition to fertility assessment, animals were visually and microscopically inspected and scored for unilateral and bilateral hydrosalpinx or cysts, and presence of other abnormalities in the reproductive system $[24,25]$. The cell permeable, irreversible pan-caspase inhibitor, N-benzyloxy-cabonyl-Val-Ala-Asp-Fluoromethyl Ketone (Z-VADFMK) (R\&D Systems, Inc. Minneapolis, MN, USA) was applied intra-vaginally at day 1 and 7 pre-infection by administering 0.02 $\mathrm{ml}$ of a $50 \mathrm{uM}$ solution in PBS per mouse under anesthesia. Control animals were similarly treated with the prescribed negative control for caspase inhibitors, Z-FA-FMK (Z-Phe-Ala-FMK). Other Fluoromethy Ketone derivatives that are specific inhibitors of caspases 1,3 and 8 used in the study were Z-WEHD-FMK (Z-Trp-Glu-His-Asp-FMK), Z-DEVD-FMK (Z-Asp-Glu-Val-AspFMK), and Z-IETD-FMK (Z-Ile-Glu-Thr-Asp-FMK), respectively.

Measurement of systemic cytokines and Antibody responses in serum of mice: The levels of systemic cytokine response in the mice were assayed by measuring Th1 (IFN- $\gamma$, TNF- $\alpha$ and T17) and Th2 (IL-5 and IL-10) cytokines in serum, at $4 \mathrm{wk}$ after infection that resulted in infertility in the mice, as previously described [26]. Briefly, sera from blood samples $(0.05 \mathrm{ml} /$ mouse $)$ were assayed for the indicated cytokines using the Bio-Plex cytokine assay kit in combination with the Bio-Plex Manager software (Bio-Rad, Hercules, CA), as previously described [26]. The mean and SD of all replicate cultures were calculated. The experiment was repeated three times. Determination of concentrations of Chlamydia-specific antibody isotypes (specifically IgG IgA) in sera measured by a standard ELISA procedure described previously [26]. Briefly, 96-well microtiter plates (Nunc Life Technologies, Rochester, NY) were coated with 10 $\mu \mathrm{g} / \mathrm{ml}$ of chlamydial antigen (i.e., UV-inactivated elementary bodies) in $100 \mu \mathrm{l}$ of PBS at $4^{\circ} \mathrm{C}$ overnight. Plates were blocked with $1 \%$ bovine serum albumin containing 5\% goat serum in PBS and $100 \mu \mathrm{l}$ of serum in twofold serial dilutions was added per well. Plates were incubated with $100 \mu \mathrm{l}$ of horseradish peroxidase-conjugated goat anti-mouse IgA or IgG isotypes (Southern Biotechnology Associates, Inc., Birmingham, AL, USA.) for $1 \mathrm{~h}$ and developed with 2,2'-azino-bis (3-ethylbenzthiazoline-6-sulfonic acid) (ABTS). The optical density was measured at $490 \mathrm{~nm}$ on a Microplate reader. Results, generated simultaneously with a standard curve, display data sets corresponding to absorbance values as mean concentrations $(\mathrm{ng} / \mathrm{ml}) \pm \mathrm{SD}$ and represent the mean values from triplicate experiments.

\section{Statistical Analysis}

Data from different experiments were analyzed and compared by performing a one- or two-tailed $t$ test or Mann-Whitney $U$ test, and the relationship between different experimental groupings was assessed by Analysis of Variance (ANOVA) using the SigmaStat software. Statistical significance was set at $\mathrm{P} \leq 0.05$.

\section{Results}

Chlamydial shedding in infected infertile mice and those protected from infertility is similar.

To better characterize the contributions of the host and pathogen to chlamydial disease, we recently reported that although both the wild-type and the plasmid-free strain of $C$. trachomatis could colonize the genital tracts of female mice, the latter failed to induce infertility as compared to the former [19]. In addition, the local genital tract application of the pan-caspase inhibitor, Z-VAD-FMK conferred protection against infertility without affecting infectivity, as assessed by shedding of chlamydiae into the cervic-vaginal vault [19]. Thus, when infected Z-VAD-FMK treated and non-treated mice were monitored for chlamydial shedding, Figure 1 shows that all animals were infected to the same degree and shed chlamydiae from their genital tracts to the same intensity during the initial 2 weeks covering the peak period of shedding after infection. However, as shown in Figure 2, treatment with the pan-caspase inhibitor, Z-VAD- 
FMK protected infected mice from infertility, as previously reported [19]. To identify the specific initiator caspase that mediate infertility, groups of mice were treated with specific inhibitors of the key initiator capases (caspase-1, -3 and -8), namely Z-WEHD-FMK, Z-DEVD-FMK, and Z-IETD-FMK, respectively, and fertility was assessed after infection. As shown in Figure 2, treatment with the specific caspase-3 inhibitor, Z-DEVD-FMK, protected infected animals from infertility comparable to the effect of the pan-caspase inhibitor, Z-VAD-FMK. Inhibition of Caspases 1 or 8 with the respective specific inhibitors was essentially ineffective against chlamydiainduced infertility, suggesting a limited role in the pathogenesis of infertility. The results corroborate previous reports that caspase- 3 is the major enzyme responsible for the cleavage inactivation of Dicer [27], the cytoplasmic processing enzyme in the biogenesis of miRNAs that control epithelial functional integrity, development and fertility-related biological activities in the reproductive system.

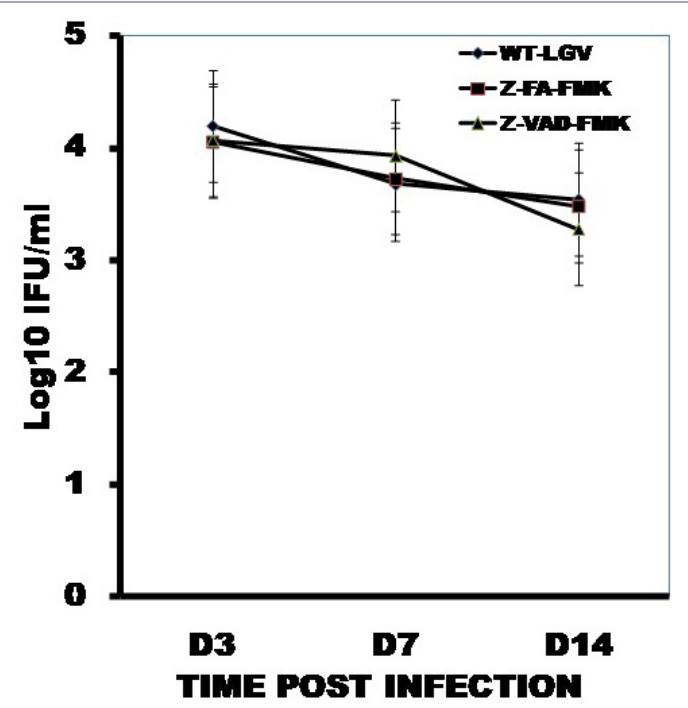

Figure 1: Comparison of the levels of chlamydial infection of mice untreated and treated with caspase inhibitor to prevent development of infertility.

Groups of female C57BL/6 mice were infected intra-vaginally with the wild-type C. trachomatis L2 (WT-CT-L2) after treatment with the pan-caspase inhibitor,
Z-VAD-FMK, the control for caspase inhibitors, Z-FA-FMK or sham-treatment Z-VAD-FMK, the control for caspase inhibitors, Z-FA-FMK or sham-treatment
with PBS. The intensity of the infection was monitored by weekly cervico-vaginal with PBS. The intensity of the infection was monitored by weekly cervico-vagina
swabbing and isolation of chlamydiae in culture as described in the Materials and Methods section for the first 3 weeks to capture the acute phase when chlamydiae shedding is optimum in this model. The plotted data are the means (SEM) from 4 independent experiments with 6 mice per experimental group.

These results further demonstrated that chlamydial infection alone without the host's contribution that involves the activation of caspase- 3 is insufficient for the induction of infertility. Therefore, the mouse genital infection model system provides a unique opportunity to evaluate and compare the host's immunologic status during an infection that leads to tubal pathologies and infection without pathologies, such as infertility that is the focus of this study.

Systemic humoral immune response in chlamydial-infected infertile and fertile hosts: We tested the hypothesis that the profile of anti-chlamydial immune response following an infection will be quantitatively or qualitatively different in infections leading to complications such as tubal pathologies or infertility when compared to the profile during infections without complications. Starting with the mouse genital infection model system, we compared the profiles of anti-chlamydial serum IgG and IgA antibodies in infected mice that developed infertility as a major complication of the genital infection and infected mice protected from the infertility due to treatment with a caspase inhibitor. Figure $3 \mathrm{a}$ shows that the concentration of total systemic (serum), specific anti-chlamydial IgG response in infected mice that did not develop infertility was approximately 4 -fold higher than that of infected mice that developed infertility (i.e., 20,000 versus

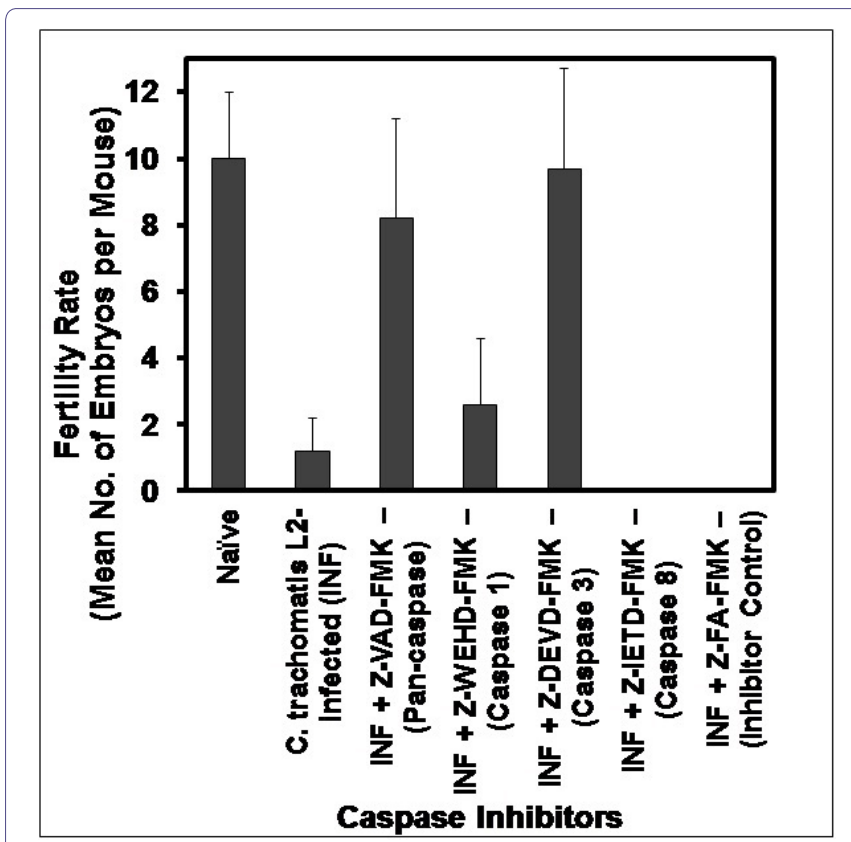

Figure 2: Caspace-3 is the main mediator of the pathologic process leading to Chlamydia-induced infertility.

Groups of female C57BL/6 mice were infected intra-vaginally with the wild-type C trachomatis L2 (WT-CT-L2) after treatment with either the pan-caspase inhibitor trachomatis L2 (WT-CT-L2) after treatment with either the pan-caspase inhibitor, Z-VAD-FMK, the specific caspase-1 inhibitor Z-WEHD-FMK, specific caspase-3 inhibitor Z-DEVD-FMK, specific caspase-8 inhibitor Z-IETD-FMK, the common control for caspase inhibitors, Z-FA-FMK or sham-treatment with PBS. The mice were mated and scored for pregnancy and number of pups, as described in the Materials and Methods section. The plotted data are the means (SEM) for groups from 3 independent experiments with 5 mice per experimental group.

$5,000 \mathrm{ng} / \mathrm{ml}$ ). Also, the concentration of total chlamydial-specific IgA in the sera of infected mice that did not develop infertility was higher than that of infected mice that developed infertility (Figure 3b). The caspase inhibitors did not alter the chlamydial-specific antibody levels in non-infected (naïve) animals. Although the role of antibodies in anti-chlamydial immunity is ancillary, the results suggested that the IgG response in animals to genital chlamydial infection may is different between infertile and fertile hosts.

Systemic cytokine response in chlamydial-infected infertile and fertile hosts: The analysis of anti-chlamydial cytokine response by Peripheral Blood Leukocytes (PBL) from infected women with and without symptoms or tubal symptoms had indicated that chlamydial control in humans is Th1-mediated, involving IFN- $\gamma$; and infections with complications are characterized by Th2 response marked with secretion of IL-4, IL-5 and IL-10 [28-31]. In the mouse genital infection model system, the inbred animals are confined in relatively hygienic conditions, compared to outbred animals or humans. Using this model system, we compared the profiles of serum cytokines in groups of chlamydial infected infertile mice and the groups protected from the infertility complication due to treatment with a caspase inhibitor. As shown in Figure 4a, beside comparable IL-2 levels, there were significantly greater levels of the Th1-type cytokines, TNF- $\alpha$, IFN- $\gamma$ and T17 in sera from infected mice that were protected from infertility than infected animals that developed infertility. Conversely, 


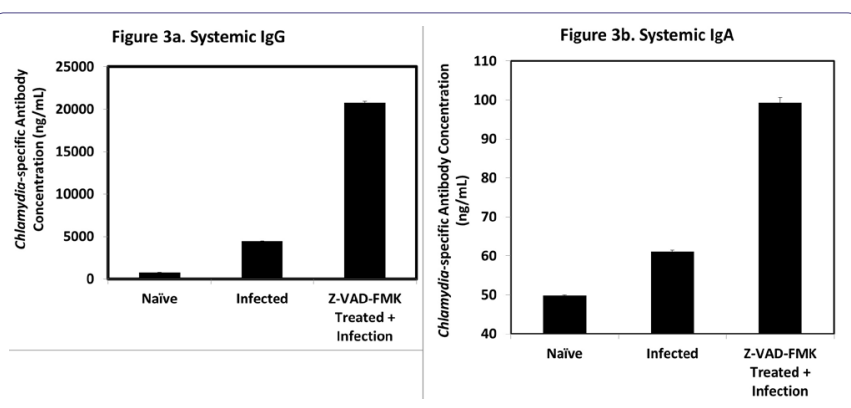

Figure 3(a,b): Systemic chlamydial-specific total $\lg G$ and $\lg A$ antibodies in

Groups of female C57BL/6 mice were infected intra-vaginally with the wild-type C. trachomatis L2 (WT-CT-L2) after treatment with the pan-caspase inhibitor C. trachomatis L2 (WT Z-VAD-FMK, or sham-treatment with PBS. Serum from final bleed after mating were assayed for the indicated chlamydial-specific total IgG (Figure 3a) and IgA (Figure $3 b$ ) by ELISA method as described in the Materials and Methods section. The mean and SD of all replicate cultures were calculated. The experiment was repeated three times.

there were significantly greater levels of the Th2-type cytokines, IL-5 and IL-10, in the serum of infected mice that developed infertility than mice protected from infertility (Figure 4b). The caspase inhibitors did not alter the cytokine levels in non-infected (naïve) animals. These results from experimental animal studies indicating that the development of complications such as infertility may affect the levels of Th1 and Th2 cytokines were corroborated by previous reports that chlamydial immunity in humans and mice is Th1 mediated [28-31].

\section{Discussions}

A better characterization of the profile of immune responses in infections with and without complications may provide molecular or immune biomarkers for prognostic indication of potentially untoward infections; this may also provide a guide in the design and evaluation of safe efficacious chlamydial vaccines. We tested the hypothesis that the anti-chlamydial immune effectors induced during infections that produce complications are qualitatively or quantitatively different from non-complicated infections. Our results are corroborated by previous reports on the analysis of anti-chlamydial cytokine response by Peripheral Blood Leukocytes (PBL) from infected women with and without tubal symptoms that indicated that chlamydial control in humans is Th1-mediated, involving IFN- $\gamma$; and that infections with complications are characterized by Th2 response marked with secretion of IL- 4 , IL- 5 and IL-10 [28-31]. Accordingly, we found significantly greater levels of the Th1-type cytokines, TNF- $\alpha$, IFN- $\gamma$ and IL-17 in sera from infected mice that were protected from infertility than infected animals that developed infertility; and there were significantly greater levels of the Th2-type cytokines, IL-5 and IL-10, in the serum of infected mice that developed infertility than mice protected from infertility. An important point to realize in these studies is that these systemic serum $T$ cell cytokine levels were obtained from confined experimental animals in sterilized hygienic conditions, which may be different in humans. In fact, the general systemic cytokine response in infected humans is not antigen or infection specific, since levels of serum cytokines are contributed by several sources that include infected cells, inflammatory cells such as macrophages, neutrophils, NK cells, dendritic cells, $\mathrm{T}$ and $\mathrm{B}$ cells. Besides, the simultaneous infection by unrelated microbes also contributes to serum cytokine levels in humans. Furthermore, unlike the scheduled infectivity and cytokine assay time-points in experimental animals, there is no synchrony

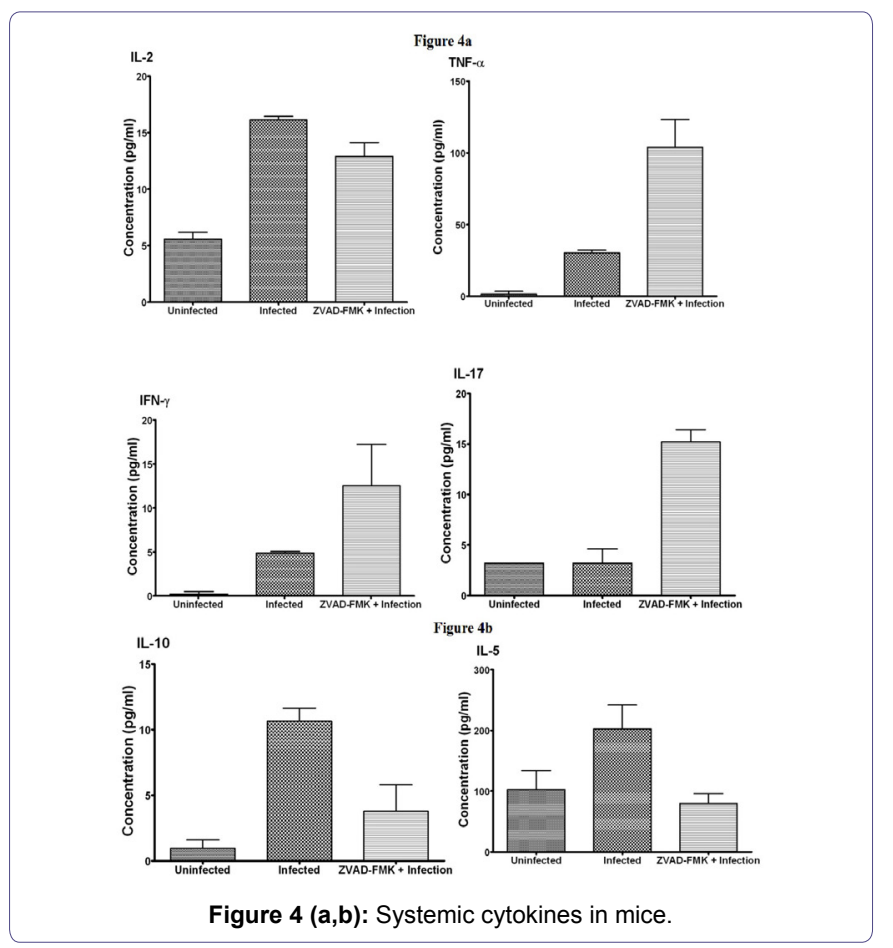

Groups of female C57BL/6 mice were infected intra-vaginally with the wild-type $C$ trachomatis L2 (WT-CT-L2) after treatment with either the pan-caspase inhibitor Z-VAD-FMK, or sham-treatment with PBS. Serum from final bleed after mating were assayed for the indicated Th1 (Figure 4a) and Th2 (Figure 4b) cytokines by were assayed for the indicated Th1 (Figure 4a) and Th2 (Figure 4b) cytokines by ELISA method as described in the Materials and Methods section. The mean and SD of all replicate cultures were calculated. The experiment was repeated three times. The mean and SD of all replicate cultures were calculated. The experiment was repeated three times.

in the infection and cytokine assay time-points among the different women recruited into this study. However, the results are similar to the cytokine measurements following antigen stimulation of PBL previously reported $[21,30]$. The key finding from this study therefore is that the results from analysis of immune profiles after genital chlamydial infection revealed that in experimental animals protection from tubal pathologies and infertility is associated with a predominantly Th1 response, which is corroborated by previous human studies using antigen-specific cytokine response by PB to chlamydial antigens $[21,30]$. Thus, the use of antigen-specific $\mathrm{T}$ cell cytokine response by PBLs to specific chlamydial antigens may represent a reliable approach to evaluate and compare chlamydialinduced cytokine responses in infections with and without pathologies in humans. This finding has important clinical implications in providing a useful prognostic biomarker for predicting the evolution of chlamydial infection and can also guide the design and evaluation of safe efficacious vaccines.

It is tempting to speculate on the clinical significance of the differential levels of immune parameters to the presence or absence of pathologies after chlamydial infections. In this respect, although the measured immune effectors do not necessarily implicate them in protective immunity or immunopathology, the identity of chlamydial antigens recognized by the cytokine-secreting $\mathrm{T}$ cells may be important for inducing protective immune responses or immunopathogenic responses, as previously suggested $[8,32,33]$. Besides, chlamydial immunity is $\mathrm{T}$ cell mediated, involving cytokine-induced anti-microbial processes that include tryptophan and essential nutrient deprivation, production of reactive nitrogen and oxygen intermediates and clearance of infected cells; while antibodies have 
Citation: Igietseme JU, Ukwade C, Ouburg S, Omosun Y, Joseph K, et al. (2015) Profile of Anti-Chlamydia Immune Responses in Complicated (Infertile) and Non-complicated (Fertile) Genital Infections. J Clin Immunol Immunother 2: 006.

supplementary role in protective immunity [4]. On the other hand, the lack of protective immunity against Chlamydia is associated with the induction of an inappropriate immune response, such as a predominantly B cell or Th2-type response [28,29]. Ultimately, it appears that chlamydial antigens that elicit the robust Th1-type cytokines in chlamydial infections without tubal pathologies or the antigens that induce the predominantly Th2-type cytokines in infections that evolve into tubal pathologies will be useful in vaccine design and screening efforts.

Finally, the finding in this study clearly suggests that the profile of the host immune response following a genital chlamydial infection could determine whether complications such as PID or Tubal Factor Infertility (TFI) will manifest in the host or not. Such a finding may provide a reliable biomarker to predict the outcome of genital chlamydial infections and therefore has important implications or key immunological parameters with prognostic value for predicting potentially complicated infections with tubal sequelae outcome if an acceptable threshold can be established for these immune parameters in humans.

\section{References}

1. Igietseme JU, Eko FO, Black CM (2003) Contemporary approaches to designing and evaluating vaccines against Chlamydia. Expert Rev Vaccines 2: 129-146.

2. Morrison RP, Caldwell HD (2002) Immunity to murine chlamydial genital infection. Infect Immun 70: 2741-2751.

3. Loomis WP, Starnbach MN (2002) T cell responses to Chlamydia Trachomatis Curr Opin Microbiol 5: 87-91.

4. Igietseme JU, Eko FO, He Q, Black CM (2004) Antibody regulation of Tcell immunity: implications for vaccine strategies against intracellular pathogens. Expert Rev Vaccines 3: 23-34.

5. Cohen CR, Koochesfahani KM, Meier AS, Shen C, Karunakaran K, et al. (2005) Immunoepidemiologic profile of Chlamydia Trachomatis infection: importance of heat-shock protein 60 and interferon-gamma. J Infect Dis 192 591-599.

6. Stephens RS (2003) The cellular paradigm of chlamydial pathogenesis. Trends Microbiol 11: 44-51

7. Rodgers AK, Budrys NM, Gong S, Wang J, Holden A, et al. (2011) Genome-wide identification of Chlamydia Trachomatis antigens associated with tubal factor infertility. Fertil Steril 96: 715-721.

8. Budrys NM, Gong S, Rodgers AK, Wang J, Louden C, et al. (2012) Chlamydia Trachomatis antigens recognized in women with tubal factor infertility, normal fertility, and acute infection. Obstet Gynecol 119: 1009-1016

9. Zeng H, Gong S, Hou S, Zou Q, Zhong G (2012) Identification of antigenspecific antibody responses associated with upper genital tract pathology in mice infected with Chlamydia muridarum. Infect Immun 80: 1098-1106.

10. Gondek DC, Roan NR, Starnbach MN (2009) T cell responses in the absence of IFN-gamma exacerbate uterine infection with Chlamydia Trachomatis. J Immunol 183: 1313-1319.

11. Lu C, Zeng H, Li Z, Lei L, Yeh IT, et al. (2012). Protective immunity against mouse upper genital tract pathology correlates with high IFN? but low IL-17 $\mathrm{T}$ cell and anti-secretion protein antibody responses induced by replicating chlamydial organisms in the airway. Vaccine 30: 475-485.

12. Matsuzaki G, Umemura M (2007) Interleukin-17 as an effector molecule of innate and acquired immunity against infections. Microbiol Immunol 51: 11391147.

13. Ley K, Smith E, Stark MA (2006) IL-17A-producing neutrophil-regulatory Tn lymphocytes. Immunol Res 34: 229-242.
14. Byrne GI (2010) Chlamydia Trachomatis strains and virulence: rethinking links to infection prevalence and disease severity. J Infect Dis 2: 126-133.

15. Bachmaier K, Neu N, de la Maza LM, Pal S, Hessel A, et al. (1999) Chlamydia infections and heart disease linked through antigenic mimicry. Science 283: $1335-1339$

16. Bachmaier K, Penninger JM (2005) Chlamydia and antigenic mimicry. Curr Top Microbiol Immunol 296: 153-163.

17. Swanborg RH, Boros DL, Whittum-Hudson JA, Hudson AP (2006) Molecular mimicry and horror autotoxicus: do chlamydial infections elicit autoimmunity? Expert Rev Mol Med 8: 1-23.

18. Cragnolini JJ, García-Medel N, de Castro JA (2009) Endogenous processing and presentation of T-cell epitopes from Chlamydia Trachomatis with relevance in HLA-B27-associated reactive arthritis. Mol Cell Proteomics 8: 1850-1859.

19. Igietseme JU, Omosun Y, Partin J, Goldstein J, He Q, et al. (2013) Prevention of Chlamydia-induced infertility by inhibition of local caspase activity. J Infect Dis 207: 1095-1104.

20. Igietseme JU, Ramsey KH, Magee DM, Williams DM, Kincy TJ, et al. (1993) Resolution of murine chlamydial genital infection by the adoptive transfer of a biovar-specific, Th1 lymphocyte clone. Reg Immunol 5: 317-324.

21. Vicetti Miguel RD, Harvey SA, LaFramboise WA, Reighard SD, Matthews DB, et al. (2013) Human female genital tract infection by the obligate intracellular bacterium Chlamydia Trachomatis elicits robust Type 2 immunity. PLoS One 8: 58565 .

22. Igietseme JU, He Q, Joseph K, Eko FO, Lyn D, et al. (2009) Role of T lymphocytes in the pathogenesis of Chlamydia disease. J Infect Dis 200: 926-934.

23. Tuffrey M, Taylor-Robinson D (1981) Progesterone as a key factor in the development of a mouse model for genital-tract infection with Chlamydia Trachomatis. FEMS Microbiol Let 12:111-115.

24. Tuffrey M, Alexander F, Woods C, Taylor-Robinson D (1992) Genetic susceptibility to chlamydial salpingitis and subsequent infertility in mice. J Reprod Fertil 95: 31-38.

25. Pal S, Hui W, Peterson EM, de la Maza LM (1998) Factors influencing the induction of infertility in a mouse model of Chlamydia Trachomatis ascending genital tract infection. J Med Microbiol 47: 599-605.

26. Eko FO, Ekong E, He Q, Black CM, Igietseme JU (2011) Induction of immune memory by a multisubunit chlamydial vaccine. Vaccine 29: 1472-1480.

27. Ghodgaonkar MM, Shah RG, Kandan-Kulangara F, Affar EB, Qi HH, et al. (2009) Abrogation of DNA vector-based RNAi during apoptosis in mammalian cells due to caspase-mediated cleavage and inactivation of Dicer-1. Cell Death Differ 16: 858-868.

28. Su L, Cao L, Zhou R, Jiang Z, Xiao K, et al. (2013) Identification of novel biomarkers for sepsis prognosis via urinary proteomic analysis using ITRAQ labeling and 2D-LC-MS/MS. PLoS One 8: e54237.

29. Wang S, Fan Y, Brunham RC, Yang X (1999) IFN-gamma knockout mice show Th2-associated delayed-type hypersensitivity and the inflammatory cells fail to localize and control chlamydial infection. Eur J Immunol 29: 37823792.

30. Batteiger BE, Xu F, Johnson RE, Rekart ML (2010) Protective immunity to Chlamydia Trachomatis genital infection: evidence from human studies. J Infect Dis 2: 178-189.

31. Cohen CR, Nguti R, Bukusi EA, Lu H, Shen C, et al. (2000) Human immunodeficiency virus type 1-infected women exhibit reduced interferon-gamma secretion after Chlamydia Trachomatis stimulation of peripheral blood lymphocytes. J Infect Dis 182: 1672-1677.

32. Karunakaran KP, Yu H, Foster LJ, Brunham RC (2010) Development of a Chlamydia Trachomatis T cell Vaccine. Hum Vaccin 6: 676-680.

33. Brunham RC, Rappuoli R (2013) Chlamydia Trachomatis control requires a vaccine. Vaccine 31: 1892-1897. 